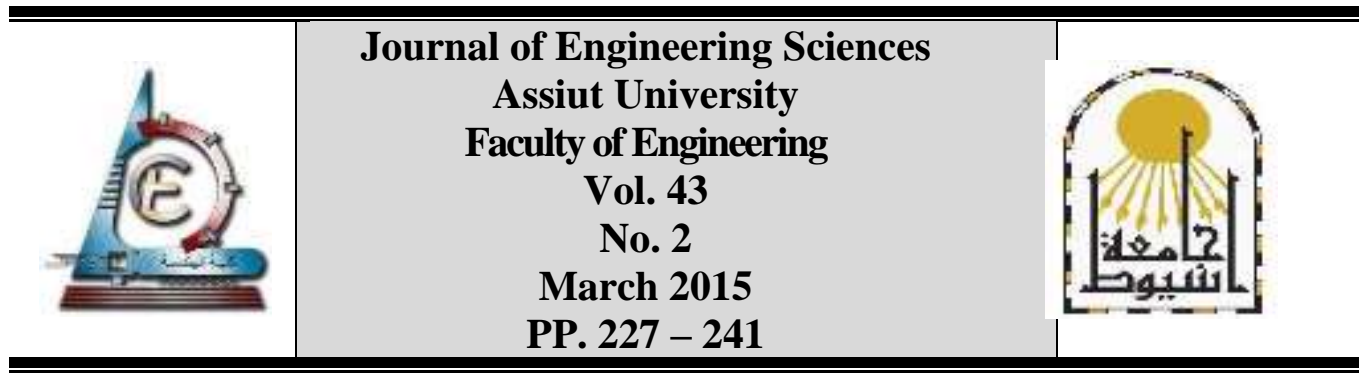

\title{
DIRECT SEQUENCE DATA SEPARATION USING ADAPTIVE BLIND DECONVOLUTION
}

\author{
Gamal M. M. Abdel Rahem, Usama Sayed Mohammed* and Khaled S. Sherif \\ Electrical Engineering Department, Faculty of Engineering, Assiut University, Assiut, Egypt
}

(Received 24 February 2015; Accepted 29 March 2015)

\begin{abstract}
Direct sequence spread spectrum (DSSS) signal is generated by modulating the pseudo random noise (PN) sequence with the data signal. The receiver must generate the same PN sequence to extract the direct sequence signal and then get the data signal. This paper presents a method to separate the PN sequence and data sequence from DSSS signal using adaptive blind deconvolution technique which utilizes the normalized cumulant of the adaptive signal. To minimize the computation burden the process gain is first estimated and is used in the adaptation process. The process gain estimator is also based on the computation of the normalized cumulant. Moreover, an efficient method that can enhance the direct-sequence spread spectrum (DSSS) signal at the receiver is introduced without complicating traditional slipping and tracking schemes. Both theoretical analysis and computer simulations verify the validity of the proposed method.
\end{abstract}

Keywords: Code division multiple access, pseudo-noise codes, maximal length, Gold, Barker, Code Tracking, Direct sequence spread spectrum, adaptive blind deconvolution, process gain, cumulant.

\section{Introduction}

In direct sequence spread spectrum (DSSS) system, the pseudo random noise (PN) sequence (wide band) is modulated with the data signal (narrow band) resulting in the wide band signal resistant to narrowband jamming and multipath fading. In the code division multiple access (CDMA) system the spreading PN codes (which are inherently orthogonal) must be typically known to the receiver to recover the incoming transmitted data. The receiver generates typical codes which must be in phase with that accompanied with the signal data. The PN code acquisitions are very tedious processes. Recently, there are too many approaches to detect the baseband DSSS signal. The conventional detecting method needs know the prior knowledge of the signal, so the detection performance decreases sharply when there is noncooperative signal. Modern spectrum estimation method can pick p spectrum characteristic information of various signals and prodigious data. But the detection performance of widely applied spectrum estimation method, such as related spectrum estimation, maximum entropy

* Corresponding author.

E-mail address: usama@aun.edu.eg 
spectral estimation and so on, deteriorates in low SNR. Gardner [1] used the related spectrum theory of cycle stable signal systematically in detecting and estimating DSSS signal but it isn't suitable for practical application because the related spectrum theory needs vast data, large amount of calculation and large memory [2-4]. G. Bruel [5] applied the autocorrelation characteristic of many sequences to detect DSSS signal. This algorithm has a good result according to the detection effect, and detection time. It is important that the G. Burel's method [5] was not available in multiuser situations. The autocorrelation function is considered a quite effective method used to detect DSSS signal in low SNR. But the performance is very bad when there is narrowband interference. Facing this problem, in [6-7] narrowband filter is used to preprocess mixed signal in the purpose of filtering out of the narrowband interference and then detecting the preprocessed mixed signal.

With the development of DS-CDMA signal processing, Blind Source Separation (BSS) technique has been applied in signal separation in the CDMA communication aboard and gets a certain progress. In DS-CDMA system, information sequences of different users remains statistically independent and the pseudo noise sequences of different users are uncorrelated, so the DS-CDMA signals satisfy the theory model of blind source separation (BSS) [8]. Some efficient methods of parameter estimation for carrier frequency [9], chip interval [10], and symbol interval [5] of the DS-CDMA signals have been developed. It is based on the ICA or the BSS to deal with the DS-CDMA signals. The BSS approach [11] does the opposite approach to detect DS-SS signal with narrowband interference based on BSS and fluctuations of autocorrelation second moment. It uses the Independent Component Analysis (ICA) to separate the aliasing signal first, and then DS-SS signal is detected by fluctuations of autocorrelation second moment. In this work [11] the noise is removed from the mixed signal first, and then DS-SS signal is detected by fluctuations of autocorrelation second moment. These approaches need large memory especially in detecting a long sequence to divide it into a window, also need a large of calculation to perform calculating of autocorrelation second order to each window. The proposed approach in this paper overcomes the high computational in the BSS approach by using the adaptive Legendre orthogonal predictor utilizing a small memory and first order transfer function. Also the predictor improves the signal-to-noise (SNR) ratio for DSSS signal. Miao Yuet. al. [12], proposed a method based on independent component analysis (ICA) to blind separation of $\mathrm{m}$-sequence from the data DS-CDMA sequence. This approach used the Eigen value decomposition (EVD) to overcome the offset that was found between the interceptor and the transmitter. However, the eigenvalues analysis is highly computational method.

This paper presents a method for recovering data sequence from DS sequence using adaptive blind deconvolution. Adaptive blind deconvolution is based on computation and minimization of normalized cumulant using higher order statistics (HOS) [13-16]. The motivation behind using the HOS are the preservation of the true phase character of signals and the fact that most real world signals are non-Gaussian and have none zero higher order spectra. Also, the higher order is quite natural when we try to analyze the nonlinearity of a system operating under a random input.

\section{Adaptive blind deconvolution based on normalized cumulant}

Deconvolution refers to the problem of determining the impulse response of the system or the input signal where the output signal is available for processing. There are two different deconvolution types. The first type is known as system identification in which the system is 
unknown, but the input is available. The second type when the system is unknown and the input signal is also unknown; this problem is referred to a blind deconvolution which is more difficult task to solve than ordinary deconvolution. To perform blind deconvolution, there are some assumptions about the statistics of the input signal. First, if the input signal is drawn from a Gaussian process (information about the system input is limited to second order statistics) then the output signal is sufficient to recover the amplitude not the phase response of the system. Second, if the input signal is non Gaussian then we shall obtain the phase information of the unknown system by extracting higher order statistics from the output signal of the system (or the received signal).

If the convolution system is a LTI system having impulse response $h(n)$ that is driven by an unknown input signal $s(n)$. The output signal $x(n)$ is available but the exciting signal $s(n)$ is not available. The adaptive blind deconvolution solves the problem of extracting $\mathrm{s}(\mathrm{n})$ from $\mathrm{x}(\mathrm{n})$. The adaptive deconvolution scheme is shown in Figure (1) where the output $x(n)$ is fed to an adaptive deconvolution with transfer function $\mathrm{g}(\mathrm{n})$. From literatures, recovering the signal and identifying the system can be achieved through maximizing the output signal normalized cumulant [21].

The output of the adaptive deconvolution is fed to a normalized cumulant computation algorithm which computes the ratiocum $6(x, x, x, x, x, y) /\left(\operatorname{cum}_{2}(x, y)\right)^{3}$, which is called the normalized cumulants [13].The computation process of normalized cumulant can be described as follows:

1. The central moments is calculated

$$
\operatorname{Mom}=\frac{1}{N} \sum_{j=1}^{N} y(j)^{p}
$$

Then the gradient of the moments is

$$
\operatorname{grad}_{\operatorname{Mom}}(p, q)=\frac{1}{N} \sum_{j=1}^{p} p^{*} y(j)^{p-1} * x(j-q)
$$

2. Computation of the Cumulant cum(p) from the recursive relation

$$
\operatorname{cum}(p)=M(p)-\sum_{j=1}^{p-1} \frac{(p-1) !}{j !(p-j-1) !} \operatorname{cum}(p-j) M(j)
$$

Where $\mathrm{M}(\mathrm{j})$ is the $\mathrm{jth}$ central moment

3. Computation of the Cumulant gradient $\operatorname{gradcum}(\mathrm{p})$ from the recursive relation:

$$
\begin{aligned}
\operatorname{grad} \_c u m(p)= & \operatorname{grad} \_\operatorname{Mom}(p)-\sum_{j=1}^{p-1} \frac{(p-1) !}{j !(p-j-1) !} * \\
& \left(\operatorname{grad} \_c u m(p-j) * M(j)+c u m(p-j) \operatorname{grad} \_\operatorname{Mom}(j)\right)
\end{aligned}
$$

4. Gradient of the Normalized Cumulant or objective cumulant is

$$
K(p, q)=\frac{\operatorname{grad} \_\operatorname{cum}(p)}{\left(\operatorname{grad} \_\operatorname{cum}(q)\right)^{p / q}}
$$


After evaluating the normalized cumulant in conjunction with its gradient it is easy to update the coefficient of the adaptive system using the adaptive algorithm.

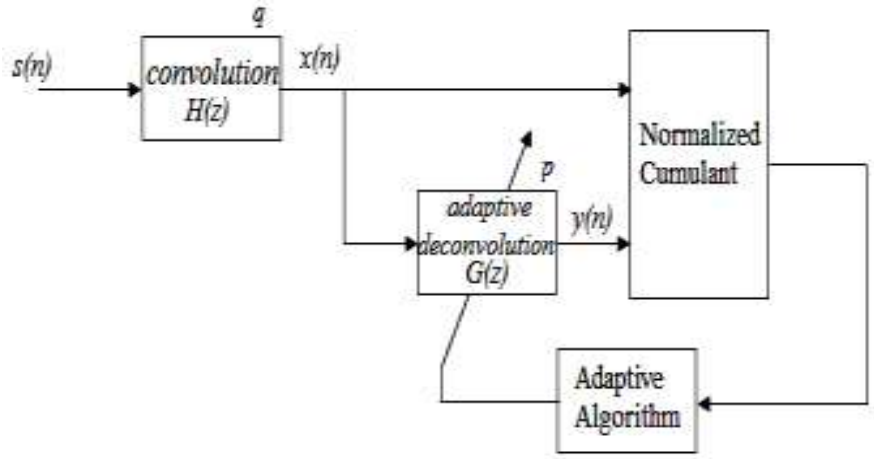

Fig. 1. General block diagram of blind deconvolution based on H.O.S

\section{The proposed data separation system based on adaptive blind deconvolution}

The proposed scheme is shown in Figure (2). It is clear that the process gain estimator is added to the adaptive blind deconvolution system. Process gain provides knowledge of the number of the blind deconvolution system coefficient (number of data sequence). The blind deconvolution process is depending on the adaptation process, where the normalized cumulant is obtained from the direct sequence signal $x_{d s}$ and the output signal from the blind deconvolution system $y_{n}$. The normalized cumulant is used to update the RLS adaptive algorithm instead of using the error signal.

Since $x_{d s}(t)=x_{d}(t) x_{n}(t)$ Where the data $x_{d}(t)$ takes values of $\pm \alpha$ and its sign is fixed during an $\mathrm{m}$ pulses of the PN codes $x_{n}(t)$. Where $\mathrm{m}$ is the process patches. The main idea of the proposed method is to divide the direct sequence data to a successive patches each patch have length $\mathrm{m}$ sample. Then, the adaptation procedures are applied for each patch to predict the adequate value of alpha $( \pm \alpha)$. The adaptation procedure is based on blind deconvolution, where the normalized cumulant of the direct sequence data is the objective function and must be minimized. The objective cumulant is given by [13-14]

$$
k(6,2)=\frac{\operatorname{cum}_{6}\left(y_{o}\right)}{\left(\operatorname{cum}_{2}\left(y_{o}\right)\right)^{3}}
$$

Where $\operatorname{cum}_{6}\left(y_{o}\right)$ is the $6^{\text {th }}$ cumulant of the output $y_{o}$ and $\operatorname{cum}_{2}\left(y_{o}\right)$ is the $2^{\text {nd }}$ cumulantof $y_{n}$, the adaptive blind deconvolution output is the PN code while the sequences of the alphas are the data sequence. For the adaptation algorithm, it is necessary to evaluate the cumulant gradient of the objective cumulant with respect to the weight to be adapted. In the following subsection, the PN sequence regeneration/predictor utilizes the orthogonal Legendre set will be illustrated. Moreover, the data patching, the process gain, and the steps of the proposed adaptive blind deconvolution system will be illustrated.

\subsection{The PN sequence regeneration/predictor utilizing the orthogonal Legendre set}

If the received data sequence $x_{d s}$ is corrupted by a noise, for example the AWGN, then the separation will not perform correctly. In this paper, to eliminate the effect of noise a predictor is used. 


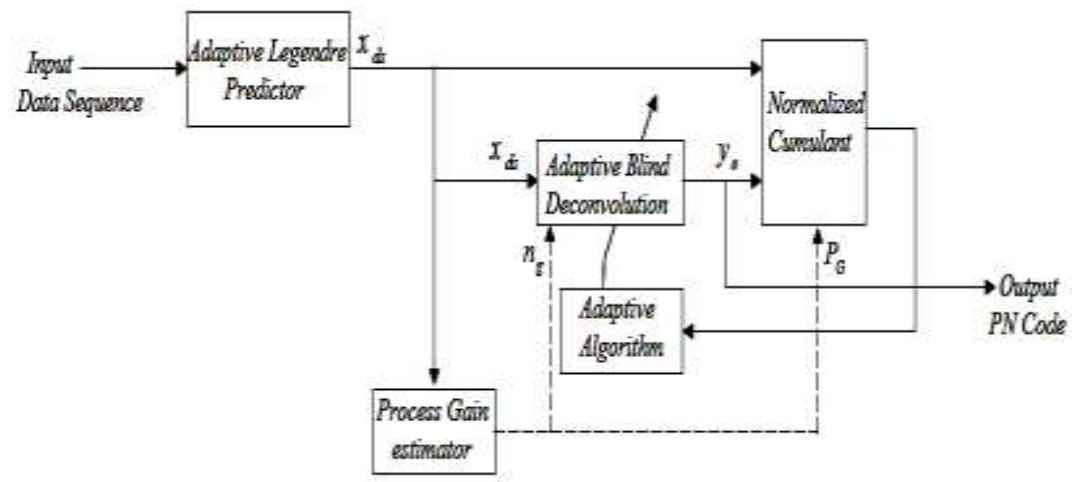

Fig. 2. The proposed adaptive Blind deconvolution system based on HOS

In case of noisy DSSS an adaptive predictor based on normalized Legendre sections is proposed to enhance the signal. In this case the output of the predictor will be identical to the original DSSS.The proposed predictor utilizes the orthogonal Legendre [17-18] section which is of the first order. This feature enables the adaptive algorithm to monitor the stability. Moreover, the orthogonal property ensures the cancelation of local minima and saddle points, which leads to fast convergence.Figure 3 shows the block diagram of the adaptive predictor [19], [20] in which nth sample can be adaptively computed by the knowledge of the past $n-m$ samples, Where $m$ is the length of predictor section. Figure 4 shows the general block diagram of the internal structure of orthogonal Legendre predictor in which nth sample can be adaptively computed by the knowledge of the past n-m samples, Where $\mathrm{m}$ is the length of predictor section. The set of the orthogonal transfer function $\mathrm{H}(\mathrm{z})$ is given by

$$
H_{j}(z)=\frac{1}{1-B z^{-1}} \prod_{j=1}^{m-1} A_{j}(z)
$$

Where $A_{j}(z)$ is an all pass function of the first order given by

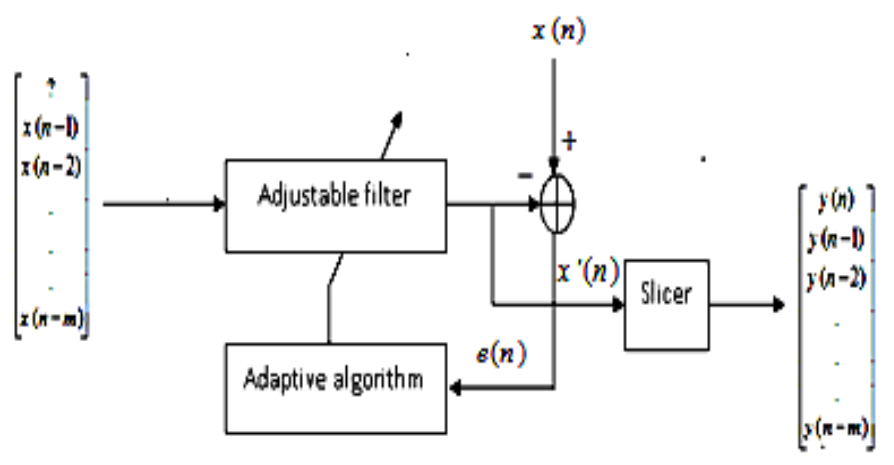

Fig. 3. General block diagram of the predictor

$$
A_{j}(z)=\frac{z^{-1}-B^{j}}{1-B^{j} z^{-1}} \quad \text { where } \quad j=1,2, \ldots, M
$$


Where $B=e^{-b}<=1$

In this class of orthogonal polynomials $b$ is taken to be positive real number less than or equal unity $(b<=1)$.Moreover, these functions satisfy the following recursive relation

$$
H_{j}(z)=\frac{1-\beta^{j-1} z^{-1}}{1-\beta^{j} z^{-1}} H_{j-1}(z)
$$

This function has the desirable simplicity and being first order makes the problem of the stability is easier. The adaptive algorithm is used to update the coefficients $\beta$ and $\mathrm{w}$.

\subsubsection{Legendre orthogonal set algorithm}

From the arrangement in Figure 4

$$
\begin{gathered}
y_{0}(n)=x(n-1) \\
y_{1}(n)=x(n)+\beta y_{1}(n-1) \\
y_{2}(n)=y_{1}(n-1)-\beta y_{1}(n)+\beta^{2} y_{2}(n-1)
\end{gathered}
$$

Then the output of the other sections in the recursive relation

$$
y_{j}(n)=y_{j-1}(n-1)-\beta^{j-1} y_{j-1}(n)+\beta^{j} y_{j}(n-1) \quad \mathrm{j}=2,3, \ldots \ldots \mathrm{m}
$$

Then the total sum of the sections or the new estimated sequence is as follows;

$$
x^{\prime}(n)=\sum_{j=1}^{m} w_{j} y_{j}(n)
$$

Then the instantaneous error signal is given by

$$
e(n)=x(n)-x^{\prime}(n)=x(n)-\sum_{j=1}^{m} w_{j} y_{j}(n)
$$

The adaptation algorithm is used in the recursive Gauss-Newton algorithm [19]

$$
\theta(n+1)=\theta(n)+\frac{e(n) R^{-1}(n+1) \nabla(n)}{\lambda+\nabla^{t}(n) R^{-1}(n) \nabla(n)}
$$

Where $\theta(n)$ and $e(n)$ are respectively the overall weight vector and the error diagonal matrix.

$$
e(n)=[\operatorname{Diag}(e(n))]
$$

$\nabla(n)$ is the gradient vector at the $n^{\text {th }}$ adaptation on a cycle. $R(k)$ isthe estimated hessian matrix at the nth adaptation on cycle. The overall gradient vector is given by

$$
\nabla(n)=\left[\nabla_{w_{1}}(n) \ldots \ldots \ldots \ldots \nabla_{w_{m}}(n) \nabla_{\beta}(n)\right]
$$

$R^{-1}(k)$ is updated directly using the inversion lemma given by [19] 


$$
R^{-1}(n+1)=\frac{1}{\lambda}\left(R^{-1}(n)-\frac{R^{-1}(n) \nabla(n) \nabla^{t}(n) R^{-1}(n)}{\lambda+\nabla^{t}(n) R^{-1}(n) \nabla(n)}\right)
$$

Since $\lambda$ is the forgetting factor.

In general, the proposed predictor can be summarized as follows:

Step 1 Start the initial value of $\beta$ and $w$.

Step 2 Calculate the output $y_{t}(n)$ and the gradient of the output $\nabla y_{t}(n)$ using a Legendre orthogonal set.

Step 3 Determine the error $e(n)$

Step 4 using the error in the update process for $\beta$ and $w$ in the adaptive technique (Legendre orthogonal set using RLS algorithm).

Step 5 Repeat the previous steps starting from step 2 until the system converges.

\subsection{Patching of the DSSS signal}
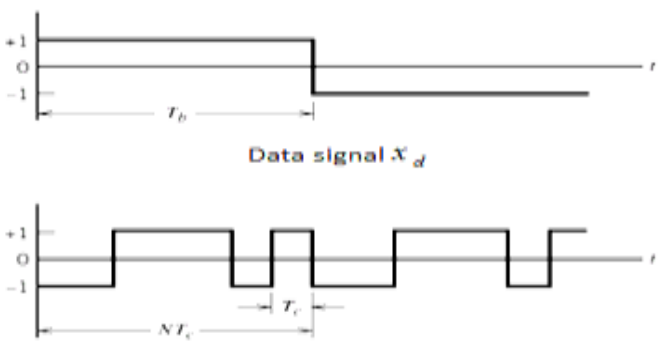

PN code sequence $x_{n}$

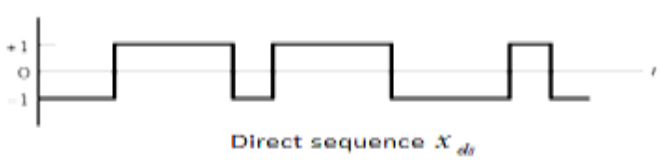

Fig. 5. DSSS signal analysis

Consider the data signal form as shown in Figure (5)

$$
\begin{aligned}
x_{d}(t) & =u(t)-2 u\left(t-T_{b}\right)+u\left(t-2 T_{b}\right) \\
& =u(t)-2 u\left(t-N T_{c}\right)+u\left(t-2 N T_{c}\right)
\end{aligned}
$$

Taking z-transform of equation (20)

$$
X_{d}(z)=\frac{1}{1-z^{-1}}\left(1-2 z^{-N}+z^{-2 N}\right)=\frac{\left(1-z^{-N}\right)^{2}}{1-z^{-1}}
$$

Where

$$
T_{b}=N T_{c}
$$


The DS-SS signal is given by

$$
x_{d s}(t)=f(t) x_{n}(t)
$$

In z-transform

$$
\begin{gathered}
X_{d s}(z)=F(z) * X_{n}(z)=\frac{\left(1-z^{-N}\right)^{2}}{1-z^{-1}} * X_{n}(z) \\
X_{d s}(z)=\frac{\left(1-z^{-N}\right)^{2}}{\left(1-z^{-1}\right)} * X_{n}(z) * \frac{\left(1-z^{-1}\right)}{\left(1-z^{-1}\right)} \\
X_{d s}(z) \frac{1}{\left(1-z^{-1}\right)}=\frac{\left(1-z^{-N}\right)^{2}}{\left(1-z^{-1}\right)^{2}} * X_{n}(z)
\end{gathered}
$$

Introducing the processing variable

$$
X_{p}(z)=\frac{1}{1-z^{-1}} X_{d s}(z)
$$

Then

$$
X_{d s}(z)=\left(1-z^{-1}\right) X_{p}(z)
$$

In the time domain

$$
x_{d s}(n)=x_{p}(n)-x_{p}(n-1)
$$

Then

$$
x_{p}(n)=x_{d s}(n)-x_{p}(n-1)
$$

\subsection{Steps of process gain estimation}

The steps of the process gain estimation can be summarized as follows:

The direct sequence $\left(x_{d s}\right)$ is passed through the auto-correlator to determine the length $\left(l_{d s}\right)$ of the sequence. The logarithm of $\left(l_{d s}\right)$ is calculated which leads to a new value $\left(l_{r}\right)$.

Number of patches $\left(n_{p}\right)$ is computed from the following relation [13]

$$
n_{p}=\log _{2}\left(\frac{l_{d s}}{2^{n_{r}}}\right) \text { where } n_{r}=l_{r}-\frac{l_{r}}{2}
$$

Start the cycle for $\left(i_{c}=1: n_{p}\right)$

Initialize the value of patches $\left.\left(x_{p}=z \operatorname{eros}\left(l_{d s}, 1\right)\right)\right)$, and $\left(x_{p}(1)=x_{d s}(1)\right)$, the rest values of the $\left(x_{p}\right)$ is computed from the following relation:

$$
x_{p}(n)=x_{d s}(n)-\beta x_{p}(n-1)
$$


where $(\beta=0.5)$ and $\left(n=2: l_{d s}\right)$.

It is clear that the equation (29) is the modified version of equation (28).

The moments and cumulant for $\left(x_{p}(n)\right)$ are computed to evaluate the normalized cumulant of $\left(x_{p}(n)\right)$. The normalized cumulant for each patch will be computed using equation (28)as follows:

$$
k_{x_{p}}(n)=\operatorname{cum}_{6}\left(x_{p}(n)\right) /\left(\operatorname{cum}_{2}\left(x_{p}(n)\right)\right)^{3}
$$

The absolute of the maximum values of the normalized cumulants of the patches $\left(\max -k_{x_{p}}\right)$ are computed. For the cycle $\left(i=1: n_{p}\right)$ compute the value $\left(w_{i}=2^{i * \max }-{ }^{k_{x}} p\right)[13]$.

The maximum value in the vector $(w)$ will represent the number of symbols $\left(n_{d}\right)$ and the process gain is computed from the ratio $\left(l_{d s} / n_{d}\right)$.

\subsection{Steps of the proposed adaptive blind deconvolution approach}

In general, the Steps of separating data from DSSS signal using the adaptive blind deconvolution can be summarized as follows:-

1) The received DSSS signal $\left(x_{d s}\right)$ will pass through the adaptive Legendre orthogonal predictor, to enhance the received data.

2) The output of the predictor will passes through the process gain estimator to select the number of blind deconvolution filter coefficients $\left(n_{d}\right)$ (number of symbols).

3) Also the output of the predictor will apply to the deconvolution filter. The coefficient of this deconvolution filter will represent the estimated data.

4) The steps of the deconvolution will be as follows:-.

4.1) The number of filter coefficient is equal to the number of the estimated data length $\left(n_{d}\right)$.

4.2)First initialize the phase of the filter coefficient (assuming it is equal to one).

4.3) Multiply the coefficient of filter with the incoming DSSS signal to calculate the output $\left(y_{o}\right)$ as shown in Figure (4).

4.4) Calculate the moments and cumulant of the output of the deconvolution filter $\left(y_{o}\right)$ and compute the objective cumulant using equation (6).

4.5) The normalized cumulant is used in the adaption process where the adaptive RLS algorithm is used to estimate the phase of the filter coefficients.

4.6) Then the filter coefficients are multiplied by the incoming data signal to recover the PN sequence.

\section{Computer simulations and results}

An adaptive simulation program based on Legendre orthogonal set predictors has been developed. In the proposed predictor, initially $\beta$ and $\mathrm{w}$ is assumed $0.5,0$; respectively, 
number of filter coefficients is 9 and the forgetting factor $\lambda$ is initially adjusted to 0.996 . However, the various feedback taps are used to generate M-sequence with a various lengths.

\section{EXP-I Separation without noise}

[1] In the case of the DSSS of length $=128$ and assuming that the original data sequence length $=2$, figure (6) illustrates the extracted data and the error signal. The error signal is calculated as the error between the extracted data and the original one

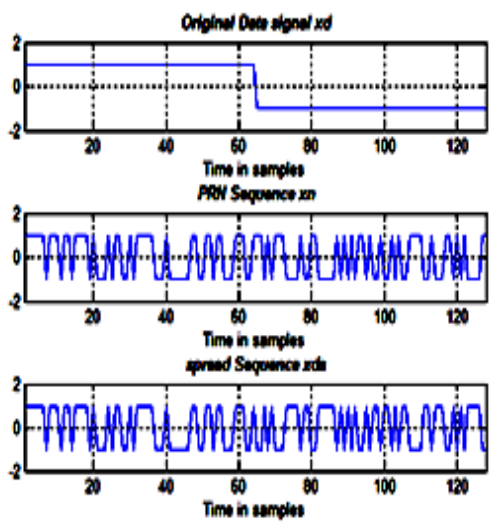

(a)

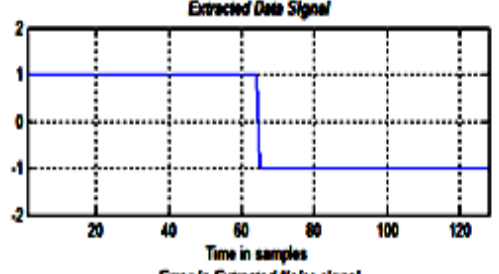

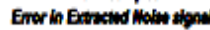

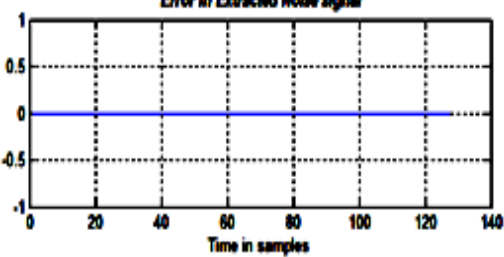

(b)

Fig. 6. (a) DSSS of length 128 from data with length 2-bit multiplied with msequencee of length 128(b) extracted data from DSSS using adaptive blinddeconvolutionand theerror signal

[2] In the case of the DSSS of length $=1024$ and assuming that the originaldata sequence length $=16$, figure $(7)$ demonstrates the performance of the proposed approach.

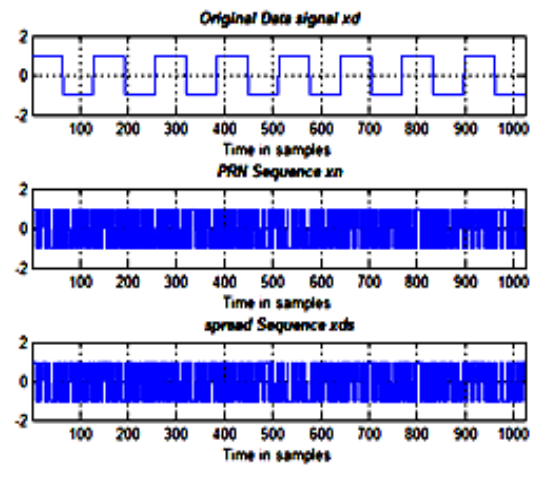

(a)
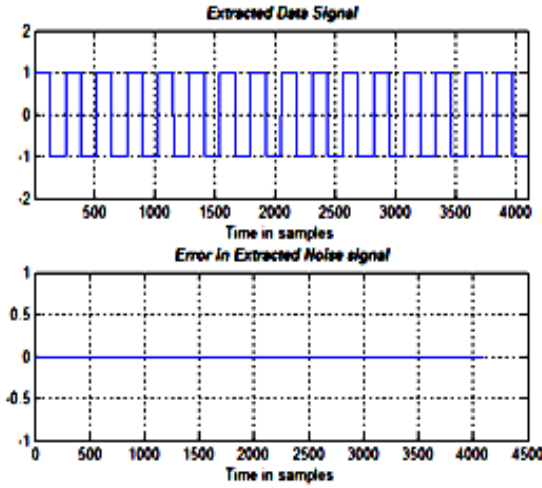

(b)

Fig. 7. (a) DSSS of length 1024 from data with length 16-bit multipliedwith msequencee of length 1024 (b) extracted data from DSSS using adaptive blind deconvolutionand theerror signal 
[3] In the case of the DSSS of length $=8129$ and assuming that the original data sequence length $=64$, figure ( 8 ) shows the system output and the error signal.

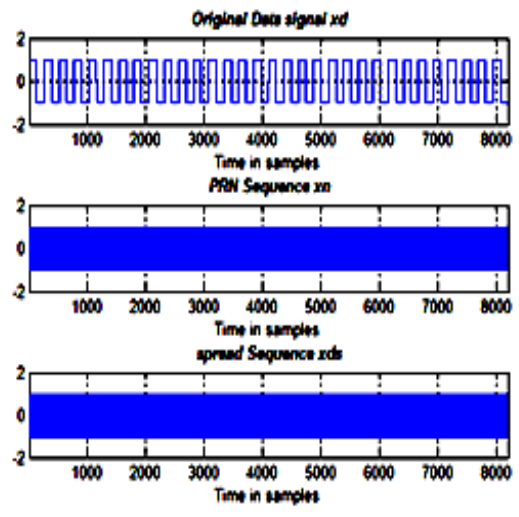

(a)

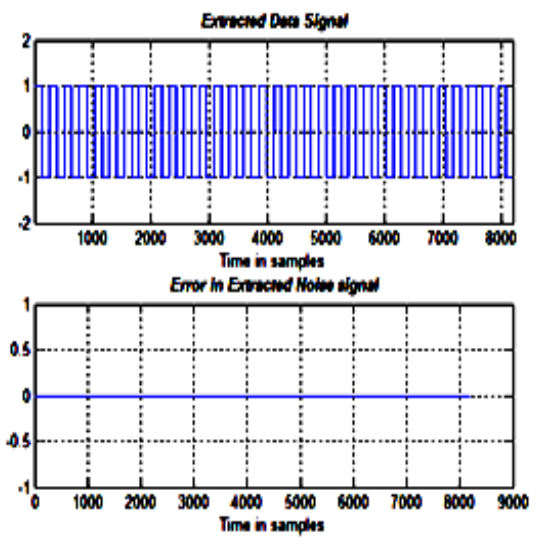

(b)

Fig. 8. (a) DSSS with length 8192 from data with length 64-bit multipliedwith msequence of length 8192. (b) extracted data from DSSS using adaptive blinddeconvolution and the error signal

From the results in Figures (6) to Figure (8), the proposed adaptive blind deconvolution provides good results in separation of the data sequence from the DSSS signal with various lengths of data. The success of the separation comes from the process gain estimator, which gave knowledge of the number of data sequence and the number of cycles for the adaptation process to estimate the exact phase of the data. Also from the proposed blind deconvolution, the PN code sequence can be exactly extracted.

\section{EXP-II Effect of the AWGN in signal separation}

To evaluate the effect of the AWGN, a discrete white Gaussian Noise is added with the critical SNR=5db for DSSS sequence with length 64 and SNR=10db for DS-SS sequence length 1024 and 4096. First the noisy direct sequence is passed through the adaptive Legendre predictor. The number predictor coefficients are selected to be 9 and the forgetting factor $(\lambda)$ is equal to 0.996 .

[1] In the case of the DSSS of length $=256$,assuming that the original data sequence length $=4$ and $\mathrm{SNR}=5 \mathrm{~dB}$, figure (9) shows the signal and the trajectory of the adaptation process in this case.

[2] In the case of the DSSS of length $=2048$, assuming that original data sequence length $=16$ and $\mathrm{SNR}=10 \mathrm{~dB}$, figure (10) shows the performance of the proposed approach.

[3] In the case of the DSSS of length $=16384$, assuming that the data sequence length $=64$ and $\mathrm{SNR}=10 \mathrm{~dB}$. figure (11) shows the performance of the proposed approach in this case.

From the simulation results in Figure (9) to Figure (11), the proposed adaptive blind deconvolution is tested in the presence of the noise. In this experiment the effect of the noise and offset is eliminated using the proposed adaptive Legendre orthogonal predictor. 
Gamal M. M. Abdel Rahem et al., Direct sequence data separation using adaptive blind .......
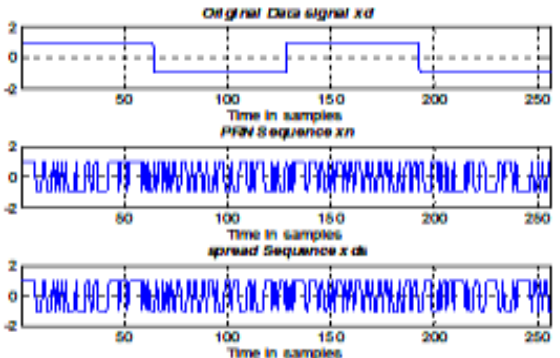

(a)
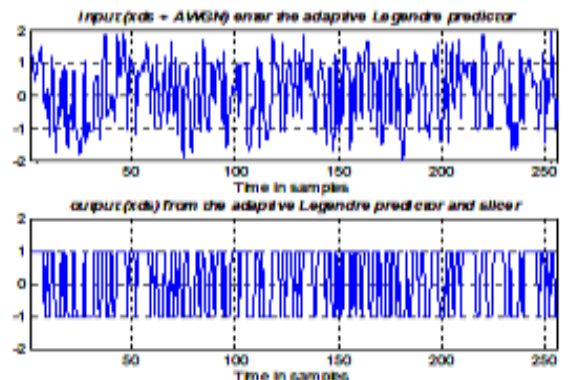

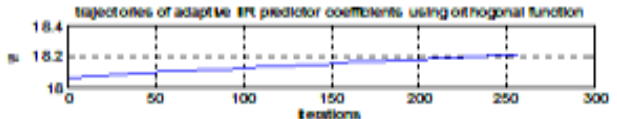

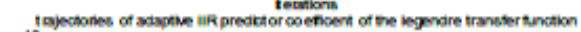

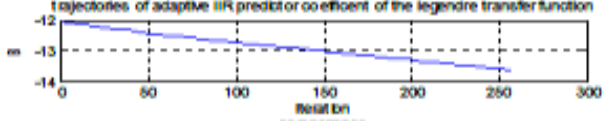

comercanoe

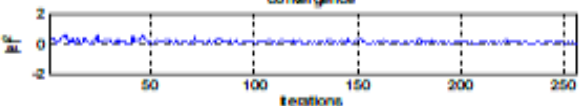

(b)
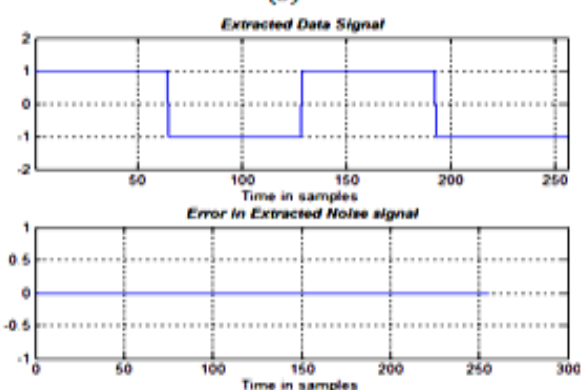

Fig. 9. (a) DSSS of data length 4 bit and PN code of length 256 (b)Trajectories of the predictors, that regeneratin the received DSSS (c) input and outputDSSS of the predictor(d) the data extracted by adaptive blind deconvolution and the error signal

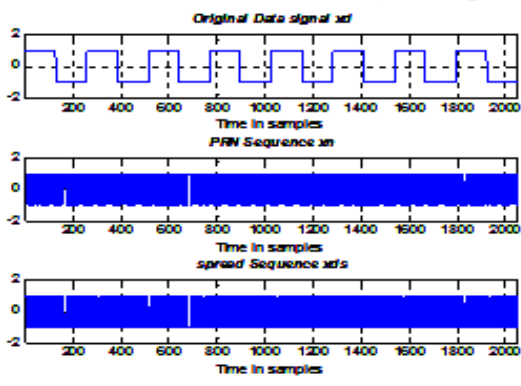

(a)

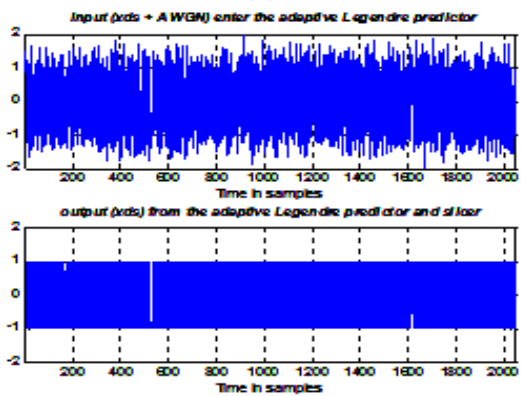

(c)
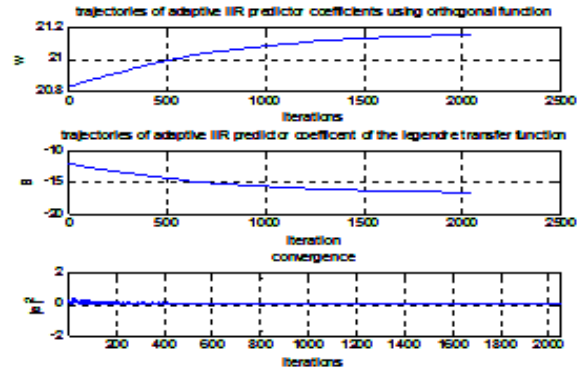

(b)

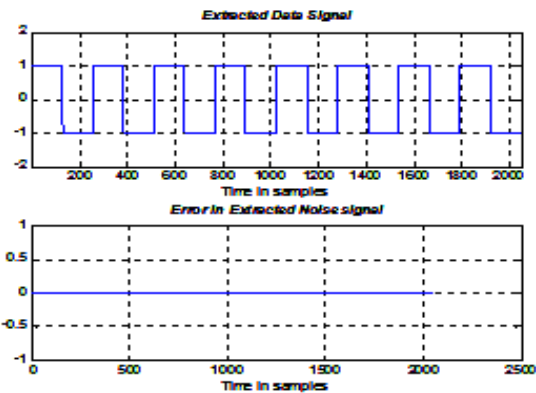

(d)

Fig. 10. (a) DSSS of data length 16 bit and PN code of length 2048 (b)Trajectories of the predictor that regeneratin the received DSSS (c) input and outputDSSS of the predictor (d) the data extracted from DSSSby adaptive blind deconvolution and the error signal. 


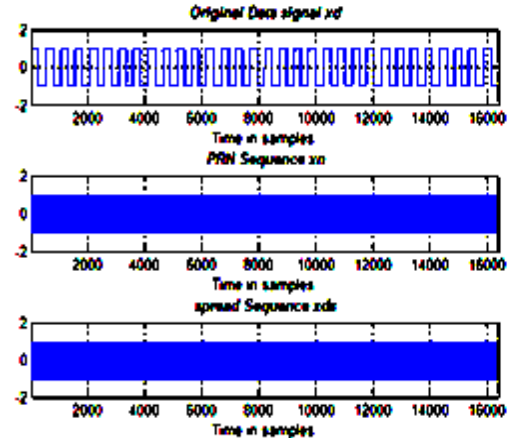

(a)

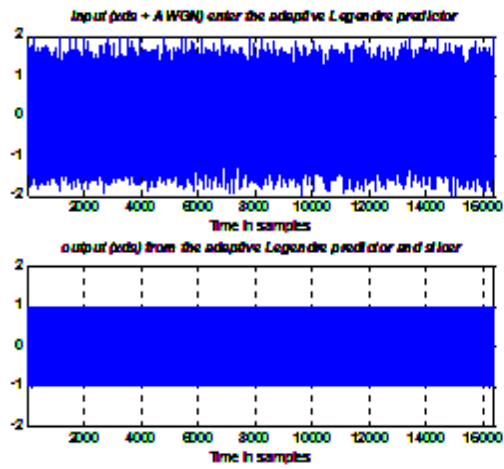

(c)

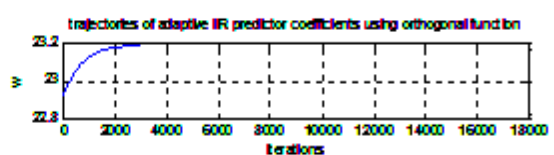

tastion:

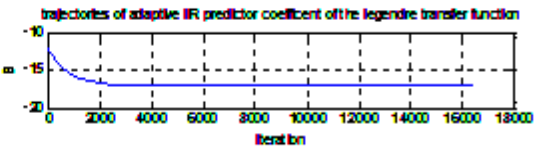
corverpence

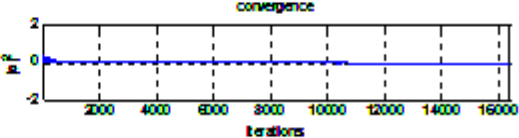

(b)

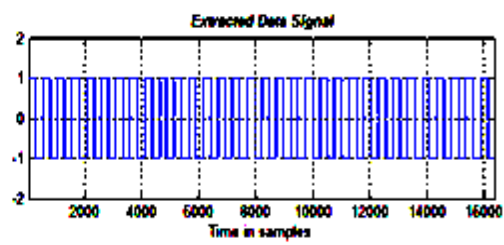

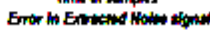

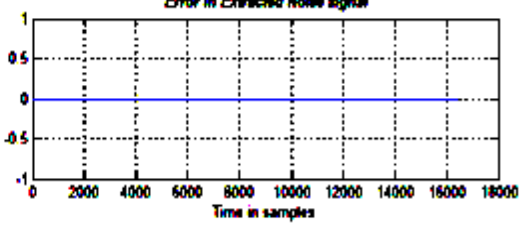

(d)

Fig. 11. (a) DS-SS of the data length 64 bit and PN code of length 16384 (b)Trajectories of predictor that regeneratin the received DS-SS (c) input and outputDS-SS from the predictor (d) the data extrated from DS-SSby adaptive blind deconvolutionand the error signal.

\section{Conclusion}

Adaptive blind deconvolution data separation of the direct-sequence spread spectrum (DSSS) sequence is proposed in this paper. The proposed approach is based on estimation of the process gain which gives us knowledge of the number of data. The process gain estimation is based on the minimization of the normalized cumulants. After the process gain estimation the DSSS sequence is divided into segments and the adaptation process is applied on each segment to detect the phase of the estimated data. Moreover, this paper presents an efficient method, based on adaptive Legendre orthogonal sections and the Recursive least squares (RLS) adaptive approach, that can regenerate/enhance the DSSS signal at the receiver without complicating traditional slipping and tracking schemes. A simulation program provides the validity of this method for blind data separation from DSSS sequence.

\section{REFERENCES}

[1] GARDNER.W.A,"Exploitation of spectral redundary in cyclostationary signals", .IEEE Trans on signal processing, vol.8, no.4, pp.14-36, 1991.

[2] Liu Tang-xing, Ji Hong-bing, "Detection of DS-SS signal using parallel filter banks and high-order cumulants", Mechatronics and automation, ICMA, vol.8, pp.2440-2444, 2007. 
[3] JIN Yan, JI Hong-bing, LUO Jun-hui, "A Cyclic-Cumulant Based Method for DS-SS Signal Detection and Parameter Estimation", Acta Electronica Sinica,vol.34, no.4, pp.634-637, 2006.

[4] Zhang Tian-qi, Zhang Wei, "A spectral method for period detection of PN sequence for weak DS-SS signals in dynamic environment",Networks Security, Wireless Communications and Trusted Computing, no.4, pp.266-269, 2009.

[5] G.Bruel, "Detection of Spread spectrum transmissions using fluctuation of correlation estimators", IEEE, ISPACS, no.11, pp.5-8, 2000.

[6] Zhang Tian-qi, Dai Shao-sheng," Method of spectrum for periodic detection of the PN sequence in the weakDS-SS signals with residual carrier", Systems Engineering and Electronics,vol.31, no.4, pp.777-781, 2009.

[7] Wanli ZUO, Ying WANG, Xin WANG, Wenyan JI, Tao PENG, "Ontology-based Filling Forms of Deep WebEntries Automatically", Journal of Computational Information Systems ,vol. 5, no.6, pp.1553-1560, 2009.

[8] O. Ekici and A. Yongacoglu, "Application of noisy independent component analysis for CDMA signal separation, Proceedings of IEEE-DSP Workshop, Vol. 3, pp. 3812-3816, July 2004.

[9] D. Hill and J. Bodie, "Carrier detection of PSK signals", IEEE Trans. on Communications, vol.49, no.3, pp.487-496, 2001.

[10] J. Yan and J. Hongbing, "A cyclic-cumulant based method for DS-SS signal detection and parameter estimation", IEEE International Symposium on Microwave, Antenna, Propagation and EMC Technologies for Wireless Communications, vol.2, 2005.

[11] H.Yang, X.Wang, Y.Zou and L.Wang, "Novel Approach to Detect the Spread Spectrum Signal and Estimate Period of PN Based on Blind Source Separation", journal of computational information systems, vol.7, no.2, pp.637-644, Feb.2011.

[12] Miao Yu, Jianzhong Chen, Lei Shen and Shiju Li,"Blind separation of DS-CDMA signals with ICA Method", journal of networks, Vol.6, No.2, February 2011.

[13] J.A. Cadzow, "Blind deconvolution via cumulant extreme", Signal processing Mag. , vol. 13, no. 3, pp. 24-42,May 1996.

[14] OfirShalvi and Ehud Weinstein, "New criteria for blind deconvolution of non minimum phase systems (channels)," IEEE Trans. Inform. Theory, vol. 36, pp. 312-321, Mar. 1990.

[15] Ofir Shalvi and Ehud Weinstein "Super Exponential Methods for Blind Deconvolution", IEEE Transactions on signal processing, vol. 39, no. 2, march 1993.

[16] D. Donoho, "On minimum entropy deconvolution," in Applied Time Series Analysis II, D. F. Findley, Ed. New York: Academic, 1981.

[17] Perez, H., and Tsuji, "A System Identification algorithm using orthogonal functions", IEEE Transaction on Signal Processing, vol. 39, pp. 752-755, Mar 1991

[18] Gamal M. Abdel Raheem, "Adaptive cancellation of noise and interference of electrocardiogram", Bulletin of faculty of engineering, AssuitUniversity, Vol. 22, No.1, January 1994.

[19] Simon Hykan,"Adaptive Filter Theory", Prentice Hall, Inc., Fourth Edition, 2002.

[20] Vaidyanathan P. P.,"The Theory of Linear Prediction", Morgan \& Claypool, 2008.

[21] Phillip A.Regalia and MamadouMboup, "Undermodeled Equalization: A Characterization of Stationary Points for Family of Blind Criteria", IEE transactions on signal processing, vol.47, no.3, pp.760-770, March 1999. 


\title{
فصل البيانات من السلسلة المباشرة في طيف الانتشار بأستخدام عملية الفصل الاعمى ذاتية التكيف الألتفافية
}

\author{
الملخص العربى
}

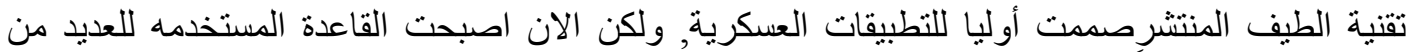

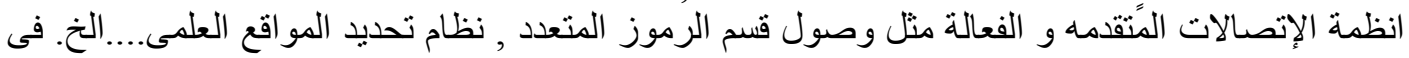

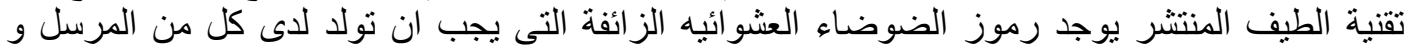

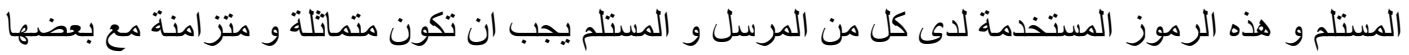

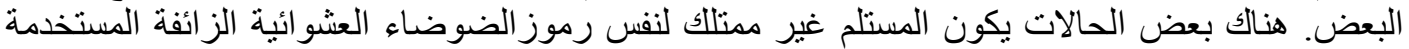

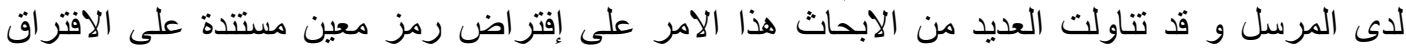

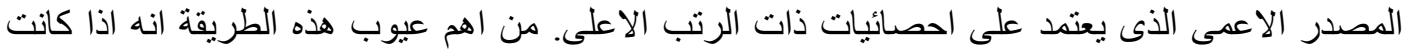

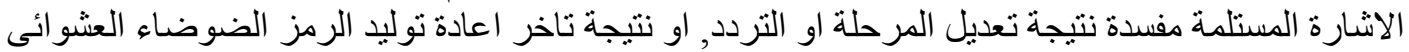

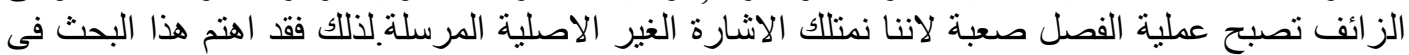

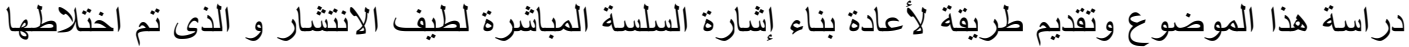

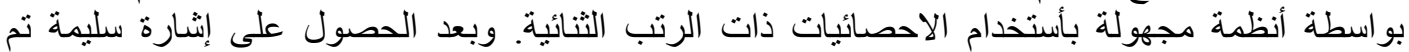

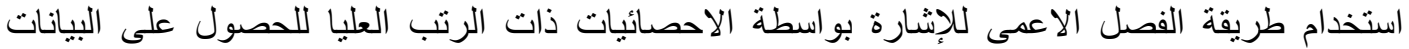

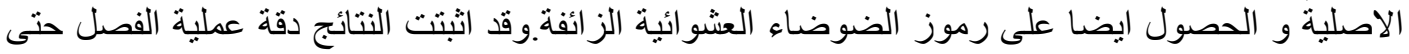
فى وجود نسبة عالية من الضوض الضوضاء. 